\title{
Observation of cyclotron resonance in an InAs/GaAs wetting layer with shallowly formed quantum dots
}

\section{Citation for published version (APA):}

Janssen, G., Goovaerts, E., Bouwen, A., Partoens, B., Daele, van, B., Zurauskiene, N., Koenraad, P. M., \& Wolter, J. H. (2003). Observation of cyclotron resonance in an InAs/GaAs wetting layer with shallowly formed quantum dots. Physical Review B, 68(4), 45329-1/6. [45329]. https://doi.org/10.1103/PhysRevB.68.045329

DOI:

10.1103/PhysRevB.68.045329

Document status and date:

Published: 01/01/2003

\section{Document Version:}

Publisher's PDF, also known as Version of Record (includes final page, issue and volume numbers)

\section{Please check the document version of this publication:}

- A submitted manuscript is the version of the article upon submission and before peer-review. There can be important differences between the submitted version and the official published version of record. People interested in the research are advised to contact the author for the final version of the publication, or visit the $\mathrm{DOI}$ to the publisher's website.

- The final author version and the galley proof are versions of the publication after peer review.

- The final published version features the final layout of the paper including the volume, issue and page numbers.

Link to publication

\section{General rights}

Copyright and moral rights for the publications made accessible in the public portal are retained by the authors and/or other copyright owners and it is a condition of accessing publications that users recognise and abide by the legal requirements associated with these rights.

- Users may download and print one copy of any publication from the public portal for the purpose of private study or research.

- You may not further distribute the material or use it for any profit-making activity or commercial gain

- You may freely distribute the URL identifying the publication in the public portal.

If the publication is distributed under the terms of Article 25fa of the Dutch Copyright Act, indicated by the "Taverne" license above, please follow below link for the End User Agreement:

www.tue.nl/taverne

Take down policy

If you believe that this document breaches copyright please contact us at:

openaccess@tue.nl

providing details and we will investigate your claim. 


\title{
Observation of cyclotron resonance in an InAs/GaAs wetting layer with shallowly formed quantum dots
}

\author{
G. Janssen, E. Goovaerts, A. Bouwen, and B. Partoens \\ Department of Physics, University of Antwerp, Universiteitsplein 1, B-2410 Antwerpen, Belgium \\ B. Van Daele \\ Department of Physics, University of Antwerp, Groenenborgerlaan 171, B-2610 Antwerpen, Belgium \\ N. Žurauskienè \\ Semiconductor Physics Institute, A. Gotauto 11, 2600 Vilnius, Lithuania \\ P. M. Koenraad and J. H. Wolter \\ Department of Physics, Eindhoven University of Technology, 5600 MB Eindhoven, The Netherlands
}

(Received 28 March 2003; published 31 July 2003)

\begin{abstract}
A thin InAs/GaAs wetting layer with shallowly formed InAs quantum dots (QD's) is investigated by means of optically detected microwave resonance spectroscopy. The absorption of $W$-band $(95 \mathrm{GHz})$ microwaves is observed via the detection of changes in the total photoluminescence intensity of the InAs QD's. A strong and anisotropic signal at low fields is ascribed to cyclotron resonance of the electron in the two-dimensional wetting layer, corresponding to an effective mass of $0.053 m_{0}$. Further microwave-induced signals at higher fields are tentatively attributed to magnetic resonance transitions between spin states of the holes confined in the shallow dots.
\end{abstract}

DOI: 10.1103/PhysRevB.68.045329

PACS number(s): 73.21.La, 76.30.-v, 76.40.+b, 78.67.Hc

\section{INTRODUCTION}

Semiconductor quantum dot (QD) structures have been shown to form ideal realizations of zero-dimensional quantum confined systems and have led to the observation of a large number of novel physical phenomena. ${ }^{1}$ Moreover, they are promising for applications in the near future, including QD-based detectors, ${ }^{2}$ lasers, ${ }^{3}$ and charge storage devices. ${ }^{4}$ In spite of the intensive research performed on InAs/GaAs selfassembled QD's, a detailed characterization of their structural and electronic properties is only recently coming into reach, applying optical spectroscopy, electron microscopy, and scanning probe microscopy. A highly sensitive approach to examine the magnetic manifolds in low-dimensional semiconductor structures is double-resonance techniques in which microwave resonance transitions are optically detected. Optically detected magnetic resonance (ODMR) spectroscopy has been advantageously applied in studies of recombination centers in semiconductors ${ }^{5}$ and of excitonic states in quantum wells, ${ }^{6}$ superlattices ${ }^{7}$ and core-shell quantum dots. ${ }^{8}$ Optically detected cyclotron resonance (ODCR) of charge carriers has been reported in bulk materials ${ }^{9}$ as well as in low-dimensional structures such as quantum wells (see, e.g., Refs. 10 and 11).

We report here on the optical detection of cyclotron and magnetic resonance transitions in a structure composed of weakly defined or shallow QD's formed as thickness and concentration fluctuations of a narrow InAs containing layer embedded in a GaAs matrix. From the observation of phonon-assisted photoluminescence (PL) measurements on the investigated structure, ${ }^{12}$ it was deduced that in the lowest exciton state, the electron and hole are localized in different regions of the shallow dots: the hole is more confined inside the dot and more compact than the electron. Furthermore, as shown by polarization-dependent PL measurements, the induced dipole is directed antiparallel to the growth direction: while the holes are localized at the apex of the dots, the electrons are at the level of the wetting layer. A similar dipole orientation was reported in Ref. 13. The separation of electron and hole partly explains the relatively long radiative lifetimes, up to $10 \mathrm{~ns}$ at $80 \mathrm{~K}$, observed for this sample. ${ }^{14}$ Optically detected microwave resonance is detected for the first time in semiconductor QD's grown by epitaxial methods, which is made possible by the exceptionally long exciton lifetimes, combined with the application of high microwave frequencies $(\approx 95 \mathrm{GHz}$, in the $W$ band) which further relaxes the lifetime requirements.

\section{SAMPLE SPECIFICATIONS}

The investigated sample was grown by molecular beam epitaxy of InAs in an amount equivalent to 2.0 monolayers (ML) on a (001)-oriented GaAs substrate as described in detail in Ref. 12. The growth process was monitored in situ by means of reflection high-energy electron diffraction (RHEED), which revealed the formation of quantum dots. Due to the relatively high growth temperature $T \geqslant 500{ }^{\circ} \mathrm{C}$ of the GaAs capping layer, the InAs material is expected to be partly diffused into this capping layer. This was confirmed by high-resolution transmission electron microscopy (TEM) measurements in a sample prepared for cross-section measurements. Instead of a sharp wetting layer with well-defined dots, an In-rich region was observed of 6-7 ML - with a total width of $\approx 2 \mathrm{~nm}-$ on top of the GaAs substrate. ${ }^{15}$ Although the TEM measurements do not reveal the presence of QD's in this structure, formation of InAs QD's is demon- 
strated by the RHEED measurements during the growth, an optical study of the exciton-phonon coupling in this sample, ${ }^{16}$ and the magnetic resonance results presented here.

\section{EXPERIMENTAL DETAILS}

Photoluminescence measurement were carried out in a liquid-helium-cooled optical cryostat, while illuminating the sample with a few milliwatts of 632.8-nm He-Ne laser light.

PL-intensity-detected ODMR spectroscopy was performed at the $W$-band microwave frequency of $\approx 95 \mathrm{GHz}$ using a custom-built Fabry-Perot cavity, ${ }^{17}$ in conjunction with a split-coil 6-T superconducting magnet (Oxford Instruments). The magnet was placed on a homebuilt rotating base for easy angular variations. A sample in the Fabry-Perot cavity can be rotated around a horizontal axis, which, together with the static magnetic field rotation around the vertical axis, permits a complete angular study. Excitation of the sample was performed through a cryostat window with the 647.1-nm light of a continuous-wave $\mathrm{Kr}^{+}$ion laser at typical incident power densities below $0.06 \mathrm{~W} / \mathrm{cm}^{2}$. The emitted light is collected via a quartz light guide and directed through a 950-nm cutoff filter, onto an avalanche Si photodiode. The microwave-induced changes in the total PL intensity were synchronously detected with the $\approx 3 \mathrm{kHz}$ on-off modulation of the applied microwaves. Experiments were performed at a temperature of $1.87 \mathrm{~K}$, using an CryoVac immersion cryostat (Troisdorf, Germany). All spectra reported here were obtained with microwaves at a frequency of $93.496 \mathrm{GHz}$, with an on-off modulation of the microwave power at $2.986 \mathrm{kHz}$.

Similar spectra were obtained with the Fabry-Perot cavity for measurements performed at $T=4.5 \mathrm{~K}$, using an Oxford Instruments helium-flow cryostat. ${ }^{18}$ Furthermore, experiments were performed at the same temperature in a commercial cylindrical cavity (Bruker) using a special fiber bundle arrangement. ${ }^{19}$

\section{RESULTS}

The PL spectrum consists of a broad peak centered at $1.31 \mathrm{eV}$ with a full width at half maximum (FWHM) of 40 $\mathrm{meV}$ (see the inset of Fig. 1). This relatively high transition energy, compared to a typical transition energy of $\approx 1.1 \mathrm{eV}$ for the lowest exciton in InAs quantum dots, ${ }^{20}$ points to the small effective size of these shallow dots. The edge of the wetting layer in this sample was determined to be around $1.345 \mathrm{eV}$ as revealed by both PL-excitation measurements and by a high-energy shoulder on the luminescence band when exciting the sample at higher densities. ${ }^{16}$

The optically detected microwave resonance spectrum, when the static magnetic field is applied parallel to the growth or $z$ axis of the sample $(B \|[001])$, is presented in Fig. 1. Two features of opposite signs are observed: at $0.178 \mathrm{~T}$ there is a negative signal, i.e., a microwave-induced decrease in the PL intensity. The width of this relatively sharp peak is $0.13 \mathrm{~T}$. The broader, positive band around 1.58 $\mathrm{T}$, with a width of approximately $0.6 \mathrm{~T}$, corresponds to a microwave-induced increase in the PL intensity. Both signals

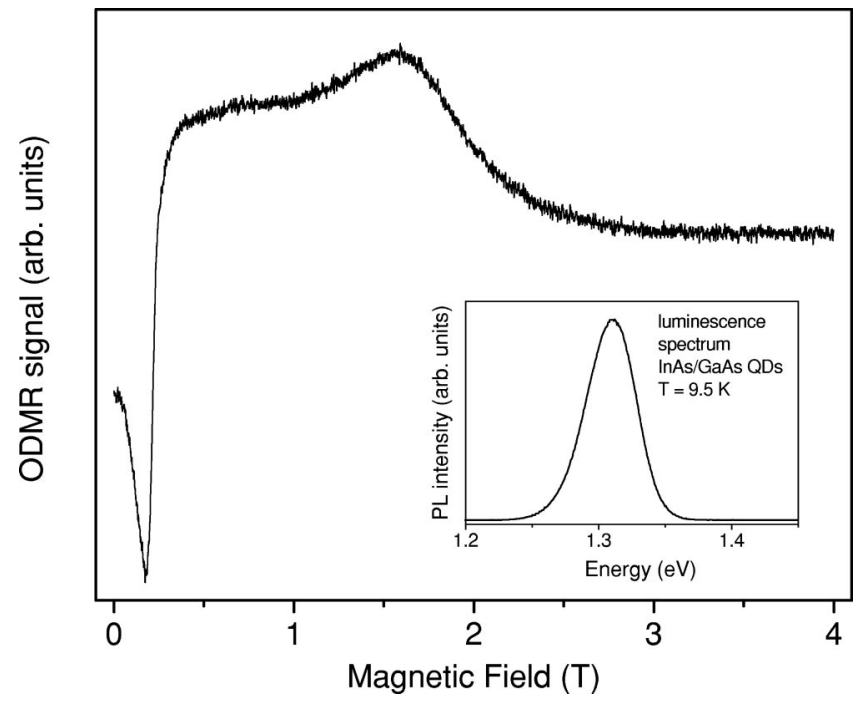

FIG. 1. Microwave spectrum measured as a modulation of the PL of the shallow InAs/GaAs QD's, shown in the inset. The static magnetic field was applied parallel to the growth axis of the sample, $B / /[001]$.

are highly anisotropic. Upon rotation of the sample or the static magnetic field direction away from the $B \|[001]$ position, the low-field line broadens while moving towards higher fields (see Fig. 2). After rotation over $30^{\circ}$ away from this parallel direction, the peak maximum is shifted to $0.25 \mathrm{~T}$ and is broadened to a width of $0.2 \mathrm{~T}$. The high-field line, however, is observed only close to the parallel direction and vanishes upon a rotation over less than $25^{\circ}$. Another anisot-

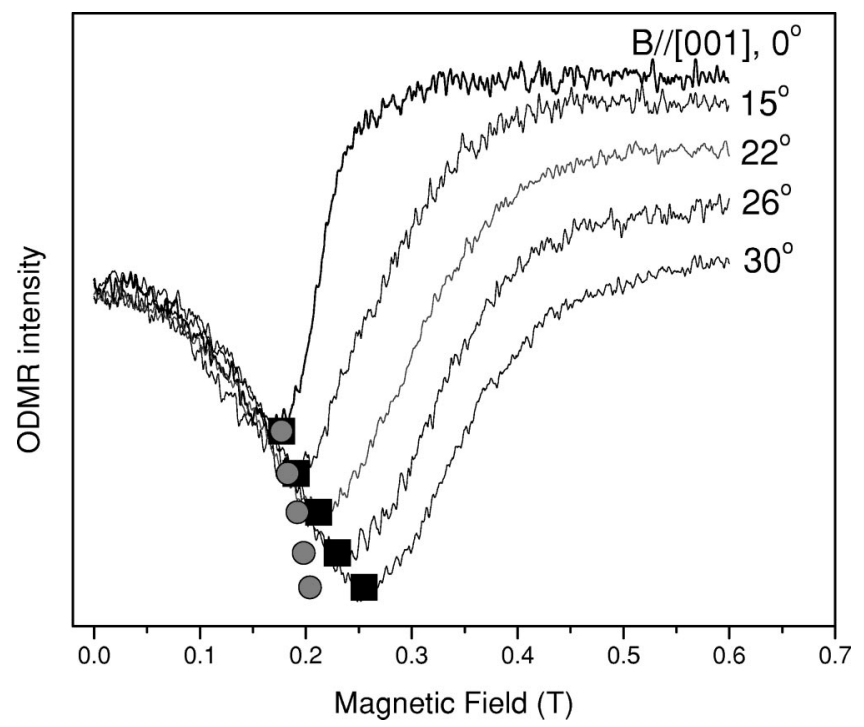

FIG. 2. Dependence of the low-field ODMR signal on the angle between the static magnetic field and the growth axis of the sample. For each angle of the tilted magnetic field, the calculated field positions are given: The circles represent the predicted field positions for the cyclotron resonance of an electron with an effective mass of $0.053 m_{0}$ in an ideal $2 \mathrm{D}$ layer. The calculated values for an electron in a parabolic quantum well in a tilted magnetic field are displayed by the squares. 


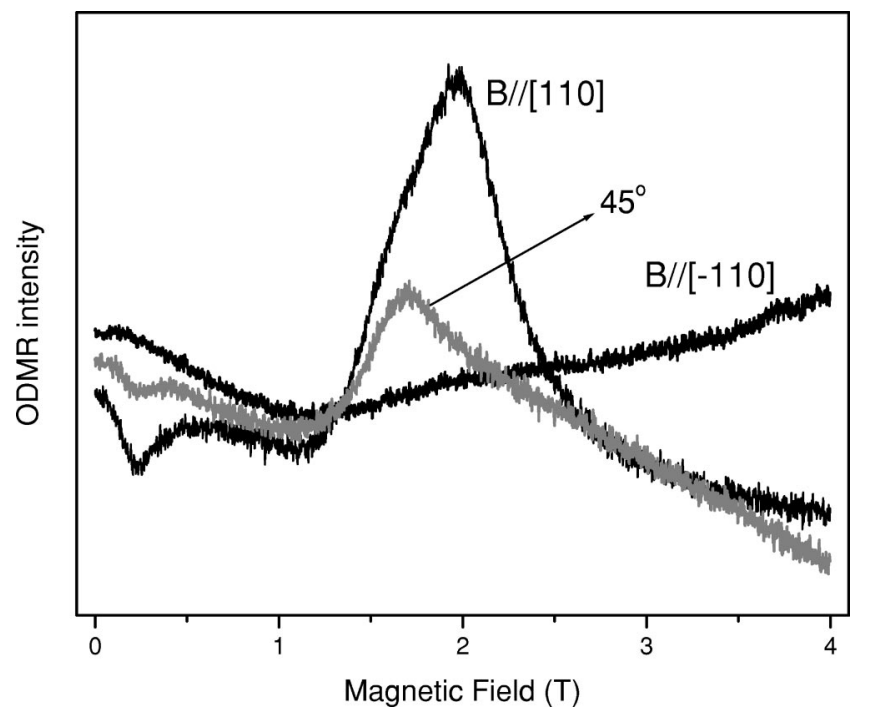

FIG. 3. Dependence of the resonance spectrum on the angle of the static magnetic field when rotating in the quantum dot plane $(B \perp[001])$.

ropy is observed when the external static magnetic field is rotated in the QD plane $(B \perp[001])$ from the [110] direction towards the [ $\overline{1} 10]$ direction, as is shown in Fig. 3. When $B$ is applied parallel to the [110] direction, two broad bands appear in the ODMR spectrum. At $0.24 \mathrm{~T}$ a negative signal is observed (width $0.29 \mathrm{~T}$ ), and around $1.96 \mathrm{~T}$ there is an asymmetric_probably composite-positive signal with a peak width of $0.8 \mathrm{~T}$. The low-field signal at $0.24 \mathrm{~T}$ decreases in intensity and is no longer observable after a rotation over $60^{\circ}$ towards the $[\overline{1} 10]$ direction. Also the high-field signal at $1.96 \mathrm{~T}$ decreases in intensity upon rotation of the magnetic field. Furthermore, the latter narrows asymmetrically, and after a rotation over $30^{\circ}$, the peak position is shifted towards 1.7 T. When $B \|[\overline{1} 10]$ no clear spectral features are observed in spite of the crystallographic equivalence with [110]. The transition of the ODMR features, from a spectrum obtained with the magnetic field direction parallel to the growth direction of the sample, towards the spectra for a field in the plane of the InAs layer, is shown in Figs. 4 and 5 for rotation from $B \|[001]$ to $[110]$ and to [110], respectively.

In order to confirm the attribution of the microwave resonances to the electronic states of the shallow QD's, measurements were performed using a cutoff filter of $950 \mathrm{~nm}$ to eliminate all optical signals at higher energy. Optically detected magnetic resonance spectroscopy was also performed on a sample grown under nearly the same conditions but without the formation of quantum dots: in this case a low growth temperature $\left(<450{ }^{\circ} \mathrm{C}\right)$ was used for deposition of the 2.0-ML InAs layer and the GaAs capping layers. At such low growth temperatures, migration of InAs on the surface is inhibited, resulting in a relatively smooth layer. This sample shows intense PL only in the region of the fundamental GaAs transition. No microwave-induced PL spectra could be detected. This is a second test demonstrating that the observed microwave resonances are directly related to the shal-

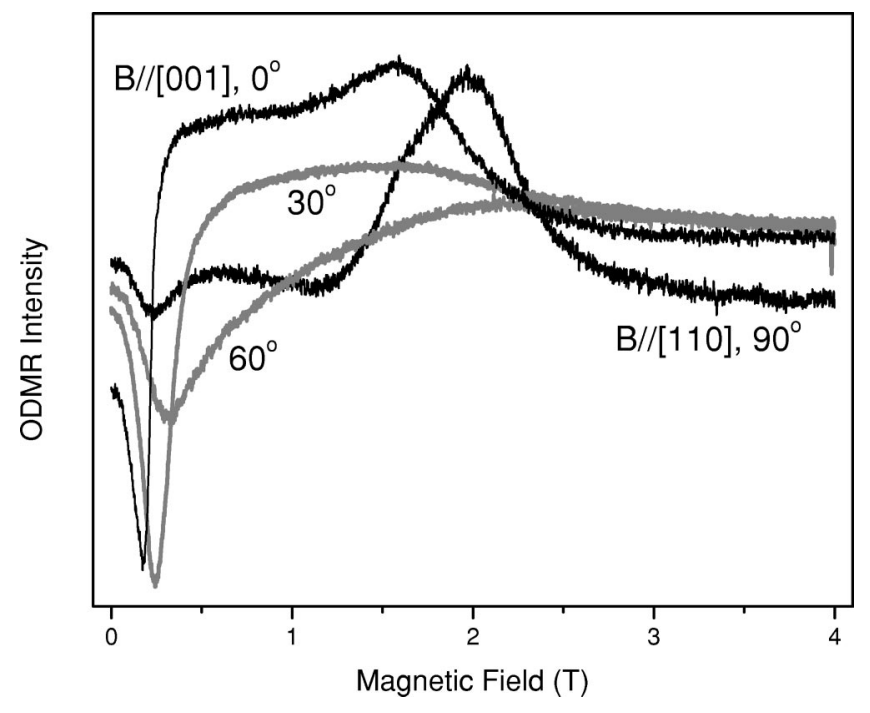

FIG. 4. Dependence of the resonance spectrum on the angle of the static magnetic field. Rotation from $B / /[001]$ towards $B / /[110]$.

low QD's, and not to spurious signals from the substrate or capping layers.

\section{DISCUSSION}

The investigated dots have a small effective size with a transition energy close to that of the wetting layer. According to previous studies, ${ }^{12}$ the holes are localized at the apex of these InAs QD's, while the electrons are close to the wetting layer. This points to a configuration where the hole is tightly confined inside the QD and the electron is not or only weakly

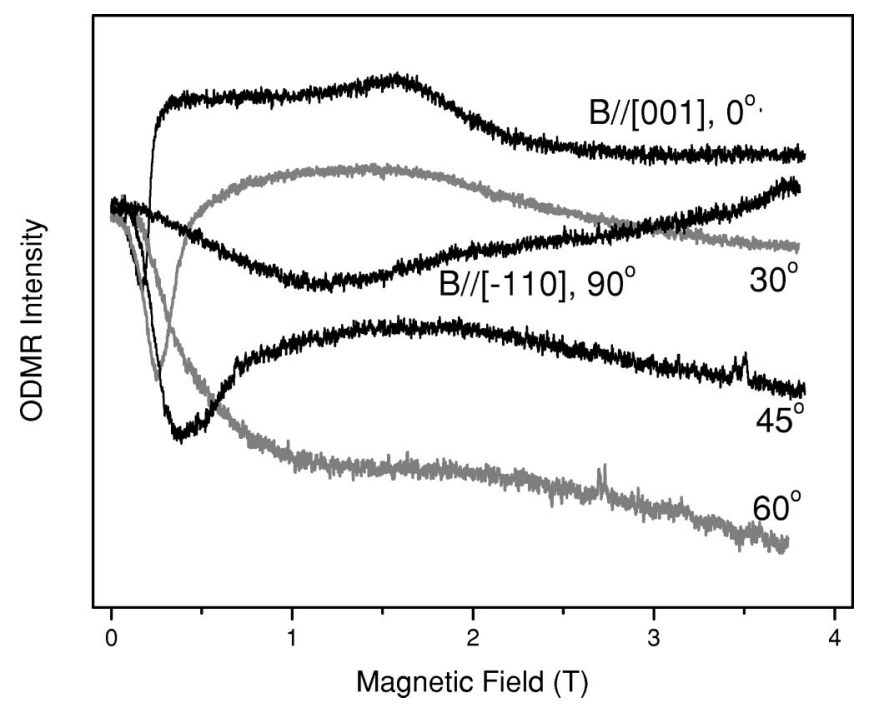

FIG. 5. Dependence of the resonance spectrum on the angle of the static magnetic field. Rotation from $B / /[001]$ towards $B / /[\overline{1} 10]$. Angular variation was obtained by rotation of the sample in the cavity around its axis. A rescaling of the intensity was applied as, in this optical geometry, the excitation intensity increases with the rotation of the sample towards the [110] direction. 


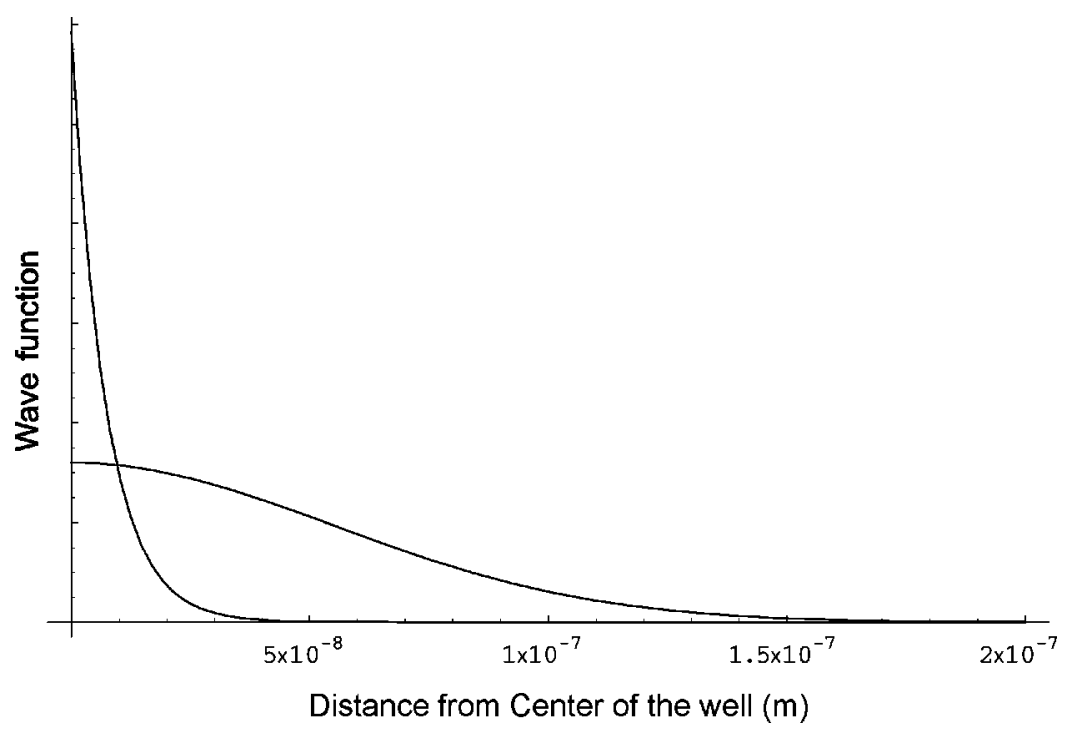

FIG. 6. The extension of the wave function of an electron with effective mass $m^{*}=0.053 m_{0}$ in a harmonic oscillator with confinement frequency $w_{0}=130 \mathrm{GHz}$ (Gaussian wave function) and that of an electron in a square well with a depth of $109 \mathrm{meV}$ and a width of $2 \mathrm{~nm}$ (exponential wave function).

bound to it and is spread out into the two-dimensional (2D) wetting layer. Various authors have reported on cyclotron resonances (CR's) of 2D-confined electrons in quantum wells and in superlattices (see, e.g., Refs. 10, 11, and 21). The low-field peak at $0.178 \mathrm{~T}$ (for $B \|[001]$ ) is attributed to CRs of the electron confined in the InAs-rich layer. This yields a value for the effective electron mass of $0.053 m_{0}$, which lies between the electron effective mass in bulk InAs $\left(0.023 m_{0}\right)$ and that in GaAs $\left(0.063 m_{0}\right){ }^{22}$ In quantumconfined structures, changes of the effective masses due to strain can be expected. Calculations of the effective masses in InAs/GaAs quantum dots predict an electron effective mass of $0.040 m_{0}$ (Ref. 23) or even $0.055 m_{0}$ (Ref. 24). An enhancement of the electron mass has been reported for InAs quantum wells and superlattices (see, e.g., Refs. 25, 11, and 26): a value of $0.050 m_{0}$ was found for the electron mass in a InAs/GaSb quantum well. ${ }^{25}$

It is worth mentioning here that we have also performed experiments on a series of well-formed InAs/GaAs QD structures, ${ }^{27}$ but all with negative results. The exciton lifetime is known to be short in these systems ( $\leqslant 1 \mathrm{~ns})$ in contrast with the investigated sample, which has been shown to possess a charge-separated exciton state ${ }^{12}$ and a much longer radiative lifetime. ${ }^{14}$

The angular dependence of the peak shows the confinement of the electron within the InAs-rich layer. In an ideal 2D system, only the magnetic field component which is perpendicular to the 2D plane induces cyclotron orbits. This yields a $1 / \cos \theta$ dependence for the peak position on the angle $\theta$ between the static magnetic field and the normal on the 0D plane. With $m^{*}$ the effective mass and $\omega_{c} / 2 \pi$ the cyclotron frequency corresponding to the transition energy of 93.486 $\mathrm{GHz}$, this dependence of the static magnetic field position is given by $B=\left(m^{*} \omega_{c}\right) /(e \cdot \cos \theta)$. In Fig. 2, for each angle $\theta$ between the static magnetic field $B$ and the [001] growth direction, the calculated field positions are plotted (circles) of an electron with an effective mass of $0.053 m_{0}$. In our case, the electron is not confined within an ideal 2D system, and thus a variation from this $1 / \cos \theta$ dependence can be expected. A similar deviation from this $1 / \cos \theta$ dependence was observed for cyclotron resonance in a GaAs/GaAlAs superlattice. $^{10}$

To take into account the finite width of the $2 \mathrm{D}$ wetting layer, the results from a parabolic quantum well in a tilted magnetic field are examined. ${ }^{29}$ The dependence of the magnetic field position on the angle $\theta$ of the static magnetic field can then be found from

$$
\omega_{\mu w} \equiv\left[\frac{1}{2}\left(\omega_{c}^{2}+\omega_{0}^{2}\right)-\frac{1}{2}\left[\omega_{c}^{4}+\omega_{0}^{4}-2 \omega_{c}^{2} \omega_{0}^{2} \cos (2 \theta)\right]^{1 / 2}\right]^{1 / 2} .
$$

In this equation $\omega_{\mu w} / 2 \pi$ corresponds to the microwave frequency of $93.486 \mathrm{GHz}, \omega_{c}$ can be found from the electron effective mass, and $\omega_{0} / 2 \pi$ is the confinement frequency of the parabolic quantum well. In Fig. 2, for each angle of the tilted magnetic field, the calculated field positions (squares) are plotted of an electron, with an effective mass of $0.053 m_{0}$, in a parabolic quantum well with a confinement frequency $\omega_{0} / 2 \pi=130 \mathrm{GHz}$. This simple theoretical model already demonstrates the influence of the finite width of the wetting layer on the field position. To examine the physical meaning of a confinement frequency of $130 \mathrm{GHz}$, a comparison was made between the extension of the wave function of an electron with effective mass $m^{*}=0.053 m_{0}$ in a harmonic oscillator with this confinement frequency of $130 \mathrm{GHz}$ and that of an electron in a square well, representing the twodimensional wetting layer. This is shown in Fig. 6. A width of $2 \mathrm{~nm}$ was used for the width of this square well, which corresponds to the width of the wetting layer as determined by TEM. The conduction-band offset of the InAs wetting layer is represented by the depth of the well. An offset of $109 \mathrm{meV}$ was extrapolated for an $\mathrm{In}_{15} \mathrm{Ga}_{85} \mathrm{As}$ quantum well. ${ }^{30}$ Taking into account the diffusion of the indium into the GaAs capping layer, an effective In concentration of only $15 \%$ was assumed. The same In concentration and a comparable InAs layer thickness were found in similar InAs/GaAs samples. ${ }^{31}$ The overlap between the two wave functions in Fig. 6 is not perfect, but one can reasonably expect the occurrence of effects like band bending, which would further 
decrease the conduction band offset and would result in an increasing similarity between the two types of wave functions. Although this is only a simple theoretical model, it supports the attribution of the observed ODMR signal to cyclotron resonance of the electron confined in the "wetting layer."

Whereas in regular cyclotron resonance experiments there is an interaction with an oscillating electric field, in our experiment the sample is placed in the center of the cavity where the electric field component of the microwaves is expected to vanish and the magnetic field component is dominant. Small transverse electric fields can, however, be expected to be present in the center of the Fabry-Perot cavity. Attempts to increase the electric field by moving the sample away from the center of the cavity result in a configuration where the cavity $Q$ factor is too low to perform reliable measurements.

The difference in linewidth, the different dependence on the magnetic field direction, and the opposite sign of the high-field signal in our spectrum for $B \|[001]$ points to a different character of this transition. As the electron is assumed to be confined outside the dot, this highly anisotropic signal is assumed to be related with a hole-spin transition of the hole in the shallow QD. The hole $g$ value $g_{h}$, corresponding to the magnetic field position of $1.58 \mathrm{~T}$, is $\left|g_{h, \|}\right|$ $=4.2{ }^{32}$ The accuracy of this value is limited by the linewidth of $0.6 \mathrm{~T}$ of the peak, which is attributed to the inhomogeneous distribution of properties of the QD's. The disappearance of the high-field signal, after rotating away from the $B \|[001]$ direction, could be explained by the fast broadening due to these inhomogeneities. The electronic structure of the holes in these shallow InAs QD's is not established; however, it is known that quantum confinement causes strong modifications of their $g$ factors. ${ }^{33,34}$
The InAs quantum dots are expected to have a pyramidal or disklike shape for which the [110] and [110] directions are equivalent. The change of the ODMR spectrum when varying the direction of the static magnetic field in this plane $(B \perp[001])$, however, points to an inhomogeneity in the plane, either in the shape of or in the strain on the shallow quantum dots. The origin of the resonance peaks, observed when the static magnetic field is applied parallel to the [110] direction, is not yet clarified and further examination will be necessary. The well-pronounced positive band at $1.96 \mathrm{~T}$, when the static magnetic field is applied parallel to the [110] direction, corresponds to a $g$ factor of $\left|g_{h, \perp}\right|=3.3 .^{32}$

In conclusion we have reported on the observation of microwave resonance transitions in a thin InAs/GaAs layer with shallowly formed quantum dots. The presence of shallow quantum dots in which electrons and holes are spatially separated was previously demonstrated by phonon-assisted PL experiments and is consistent with our optically detected magnetic resonance measurements. Indeed, the cyclotron motion in the 2-nm InAs-rich layer is detected and described in a model of partial 2D confinement. Additional features in the spectra are attributed to magnetic resonances of the holes localized in the shallow QD's.

\section{ACKNOWLEDGMENTS}

This work was supported by the National Fund for Scientific Research (FWO-Flanders, group research projects G.0110.96 and G.0409.02). The TEM measurements were performed in the framework of an Inter University Attraction Pole project (IAP-V). One of us (B.V.D.) thanks the FWO for support.
${ }^{1}$ For a review, see, e.g., D. Bimberg, M. Grundmann, and N. N Ledentsov, Quantum Dot Heterostructures (Wiley, Chichester, 1999).

${ }^{2}$ S. Sauvage, P. Boucaud, F. H. Julien, J. M. Gerard, and V. Thierry-Mieg, Appl. Phys. Lett. 71, 2785 (1997).

${ }^{3}$ K. Eberl, Phys. World 10, 47 (1997); N. N. Ledentsov, V. M. Ustinov, V. A. Shchukin, P. S. Kop'ev, Z.I. Alferov, and D. Bimberg, Semiconductors 32, 343 (1998).

${ }^{4}$ G. Abstreiter et al., Jpn. J. Appl. Phys., Part 1 38, 449 (1999); G. Yusa, and H. Sakaki, Appl. Phys. Lett. 70, 345 (1997).

${ }^{5}$ See, e.g., J. M. Trombetta and T. A. Kennedy, Phys. Rev. B 48, 17031 (1993).

${ }^{6}$ See, e.g., H. W. van Kesteren, E. C. Cosman, W. A. J. A. van der Poel, and C. T. Foxon, Phys. Rev. B 41, 5283 (1990); C. Y. Hu, W. Ossau, D. R. Yakovlev, G. Landwehr, T. Wojtowicz, G. Karczewski, and J. Kossut, ibid. 58, R1766 (1998).

${ }^{7}$ See, e.g., N. G. Romanov, P. G. Baranov, I. V. Mashkov, P. Lavallard, and R. Planel, Solid-State Electron. 37, 911 (1994); E. R. Glaser, T. A. Kennedy, D. J. Godbey, P. E. Thompson, K. L. Wang, and C. H. Chern, Phys. Rev. B 47, 1305 (1993).

${ }^{8}$ E. Lifschitz, I. Dag, I. D. Litvin, and G. Hodes, J. Phys. Chem. B
102, 9245 (1998).

${ }^{9}$ See, e.g., R. Romestein and C. Weisbuch, Phys. Rev. Lett. 45, 2067 (1980); K. Sotah, H. Nakata, and T. Ohyama, Phys. Status Solidi B 210, 361 (1998).

${ }^{10}$ B. C. Cavenett and E. J. Pakulis, Phys. Rev. B 32, 8449 (1985).

${ }^{11}$ M. J. Yang et al., Phys. Rev. B 47, 1691 (1993).

${ }^{12}$ A. W. E. Minnaert, A. Yu. Silov, W. van der Vleuten, J. E. M. Haverkort, and J. H. Wolter, Phys. Rev. B 63, 075303 (2001).

${ }^{13}$ P. W. Fry, I. E. Itskevich, D. J. Mowbray, M. S. Skolnick, J. J. Finley, J. A. Barker, E. P. O'Reilly, L. R. Wilson, I. A. Larkin, P. A. Maksym, M. Hopkinson, M. Al-Khafaji, J. P. R. David, A. G. Cullis, G. Hill, and J. C. Clark, Phys. Rev. Lett. 84, 733 (2000).

${ }^{14}$ N. Zurauskiene, S. Marcinkevicius, G. Janssen, E. Goovaerts, A. Bouwen, P. M. Koenraad, and J. H. Wolter, Mater. Sci. Forum 384-385, 31 (2002).

${ }^{15}$ Note that the distance between the atomic In layers, deduced from the analysis of the the TEM images in this In-rich layer, corresponds to a nearly pure InAs composition. This however is in contradiction with the calibrated amount of deposited material and the measured layer thickness of $2 \mathrm{~nm}$. Also, detailed scanning tuneling microscopy (STM) measurements performed on 
similar samples point to concentrations below 20\%. Further investigation of the interpretation of such cross-section TEM images is needed.

${ }^{16}$ A. Minnaert, Ph.D. thesis, University of Eindhoven, 2001.

${ }^{17}$ J. Allgeier, J. A. J. M. Disselhorst, R. T. Weber, W. Th. Wenckebach, and J. Schmidt, in Modern Pulsed and Continuous-Wave Electron Spin Resonance, edited by L. Kevan and M. K. Bowman (Wiley, New York, 1990), pp. 267-283.

${ }^{18}$ N. Zurauskiene, G. Janssen, E. Goovaerts, A. Bouwen, D. Schoemaker, P. M. Koenraad, and J. H. Wolter, Phys. Status Solidi B 224, 551 (2001).

${ }^{19}$ G. Janssen, A. Bouwen, P. Casteels, and E. Goovaerts, Rev. Sci. Instrum. 72, 4295 (2001).

${ }^{20}$ R. Heitz, M. Veit, N. N. Ledenstov, A. Hoffmann, D. Bimberg, V. M. Ustinov, P. S. Kopev, and Z. I. Alferov, Phys. Rev. B 56, 10435 (1997); M. J. Steer, D. J. Mowbray, W. R. Tribe, M. S. Skolnick, M. D. Sturge, M. Hopkinson, A. G. Cullis, C. R. Whitehouse, and R. Murray, ibid. 54, 17738 (1996).

${ }^{21}$ Y. Guldner, J. P. Vieren, P. Voisin, M. Voos, L. L. Chang, and L. Esaki, Phys. Rev. Lett. 45, 1719 (1980).

${ }^{22}$ M. Levinshtein, S. Rumyantsev, and M. Shur, Handbook Series on Semiconductor Parameters (World Scientific, Singapore, 1999).

${ }^{23}$ M. A. Cusack, P. R. Briddon, and M. Jaros, Phys. Rev. B 56, 4047 (1997).

${ }^{24}$ I. E. Itskevich, M. Henini, H. A. Carmona, L. Eaves, P. C. Main, D. K. Maude, and J. C. Portal, Appl. Phys. Lett. 70, 505 (1997).

${ }^{25}$ T. P. Smith III and F. F. Fang, Phys. Rev. B 35, 7729 (1987).

${ }^{26}$ R. J. Nicholas, Y. Shimamoto, Y. Imanaka, N. Miura, N. J. Ma- son, and P. J. Walker, Solid-State Electron. 40, 181 (1996).

${ }^{27}$ Experiments were performed on a similar InAs/GaAs structure, also with small dots, but with a lower growth temperature of the capping layer. A clear dot formation in this structure is visible from STM and AFM measurements (Ref. 31). Also a series of larger, well-defined InAs QD's in GaAs were examined, like, e.g., the 20-nm-wide dots described in Refs. 13 and 28. Furthermore, we have examined $n$-type and $p$-type InAs/GaAs stuctures grown by P. Basmaji (São Paulo, Brazil), a InAs/GaAs structure grown at the Max-Planck-Institut (Stuttgart, Germany), and a similar structure provided by the Technical University of Berlin.

${ }^{28}$ D. M. Bruls, J. W. A. M. Vugs, P. M. Koenraad, H. W. M. Salemink, J. H. Wolter, M. Hopkinson, M. S. Skolnick, F. Long, and S. P. A. Gill, Appl. Phys. Lett. 81, 1708 (2002).

${ }^{29}$ L. Brey, N. F. Johnson, and B. I. Halperin, Phys. Rev. B 40, 10647 (1989).

${ }^{30}$ G. L. Bir and G. E. Pikus, Symmetry and Strain-Induced Effects in Semiconductors (Wiley, New York, 1974). And for the parameters, O. Stier, M. Grundmann, and D. Bimberg, Phys. Rev. B 59, 5688 (1999); I. Vurgaftman, J. R. Meyer, and L. R. RamMohan, J. Appl. Phys. 89, 5815 (2001).

${ }^{31}$ P. Offermans, P. M. Koenraad, and J. H. Wolter (unpublished).

${ }^{32}$ There is a deviation from the $g$ values reported in Ref. 18 where a zero-field splitting of $45 \mu \mathrm{eV}$ was taken into account to calculate the $g$ values.

${ }^{33}$ See, e.g., A. A. Kiselev, E. L. Ivchenko, and U. Rossler, Phys. Rev. B 58, 16353 (1998).

${ }^{34}$ M. Bayer, O. Stern, A. Kuther, and A. Forchel, Phys. Rev. B 61, 7273 (2000). 